\title{
VOLUME HOLOGRAPHIC MATERIAL FOR RED SPECTRAL RANGE BASED ON POLYMER WITH ANTHRACENE SIDE GROUPS
}

\author{
U. V. Mahilny, ${ }^{\text {a,* }}$ A. I. Stankevich, ${ }^{\text {a }}$ E. A. Khramtsou, ${ }^{\text {a,b }}$ \\ and A. P. Shkadarevich ${ }^{\mathrm{b}}$
}

UDC 539.22

The possibility of volumetric phase recording based on photo-oxidation of the side anthracene groups of a new polymer with a glass transition temperature of $338 \mathrm{~K}$ by oxygen that enters the layer across the boundary with the atmospheric medium, was established experimentally. Volumetric holographic gratings with a maximum diffraction efficiency of 2.5-9.5\% were recorded with the radiation from a He-Ne laser $(\lambda=633 \mathrm{~nm})$ in layers 5-20- $\mu$ m thick. It was shown that in layers thicker than $10 \mu \mathrm{m}$ the diffraction efficiency is limited by saturation of growth in the modulation depth of the optical path difference. This effect is due to increase in the radius of the photorefraction sphere around the photosensitizer molecule with the increased photoconversion of the anthracene groups, which lowers the resolution of the holographic grating and its effective thickness.

Keywords: sensitized photo-oxidation, optical phase recording, volumetric holograms.

Introduction. Photo-oxidation of anthracene in a polymeric medium forms the basis of the operation of the volume holographic medium reoxane, used for recording volume phase holograms and developed at the S. I. Vavilov State Optical Institute at the end of the 1970s [1-4]. The product of this photoreaction (transannular anthracene peroxide) has lower molecular refraction than the initial anthracene, and this reduces the refractive index of the material as a result of phototransformation. The photoreaction develops on account of transfer of oxygen molecules with which the photo layer was previously saturated into an electronically saturated singlet state by transfer of energy from a sensitizer. Various dyes that have absorption in the whole visible region of the spectrum can be used as sensitizer, and this makes it possible to vary the region of spectral sensitivity of the material in this range [3]. The need to saturate the reoxane layers with oxygen at high pressure creates technical difficulties in the application of the material and limits the time for which its photosensitivity is maintained. Another disadvantage of the photorecording material is the mobility of both the anthracene molecules and the molecules of its peroxide in the polymeric matrix, which reduces the stability of the recorded holograms with time [5]. It is possible in practice to stop translational diffusion of the anthracene molecules and their photooxidation products if the former are chemically bonded to the macromolecules during synthesis of the polymer. Here it is better to include the anthracene molecules in the side chain in order to avoid the formation of rigid-chain polymers with excessively high glass transition temperature and a tendency to crystallize. A material with a low glass transition point and, as a consequence, significant permeability for atmospheric oxygen [6] can secure photo-oxidation of the anthracene structures in layers of limited thickness without previous saturation with oxygen at high pressure.

The aim of the present work was to confirm experimentally the assumptions that were made and to demonstrate the possibility of recording holographic gratings volumetrically by photo-oxidation of the anthracene side groups in the synthesized polymer with atmospheric oxygen.

Experimental. The polymer base of the material was a copolymer containing units with side fragments in the form of monosubstituted anthracene and photo neutral units that lower the glass transition point $\left(T_{c}\right)$. During selection of the copolymer the $T_{c}$ value of the anthracene-containing homopolymer was determined by three methods. In [7], the following empirical equation was proposed:

\footnotetext{
* To whom correspondence should be addressed.
}

${ }^{\mathrm{a}}$ Belarusian State University, Minsk, 220030, Belarus; email: mogilny@bsu.by; ${ }^{\mathrm{b}}$ Unitary Enterprise "STC "LEMT" of the BelOMO," Minsk, 220114, Belarus; email: hramtsovea@gmail.com. Translated from Zhurnal Prikladnoi Spektroskopii, Vol. 88, No. 1, pp. 159-165, January-February, 2021. Original article submitted October 13, 2020. 


$$
\log T_{c}=\frac{d}{M} \sum_{i} K_{i}^{\prime}-A
$$

where $d$ is the density of the polymer measured at $20^{\circ} \mathrm{C} ; M$ is the molecular mass of the repeating unit; $K_{i}^{\prime}$ represents the increments of the atoms and bonds contained in the repeating unit; $A$ is a constant for a specific series of polymers. By substituting $K_{i}^{\prime}$ from [7] for a series of polymethacrylates we obtain the required parameters for Eq. (1) and $T_{c}=509 \mathrm{~K}$.

In the second version a universal equation linking the glass transition point $T_{c}$ to a series of parameters for the chemical structure of the repeating unit in the macromolecules, which does not require knowledge of the density, was used [8]:

$$
\log T_{c}=\frac{\sum_{i} K_{i}^{*}}{N_{A} \sum_{i} \Delta V_{i}}+A,
$$

where $\sum_{i} K_{i}^{*}$ is an additive value with volume dimensions; $\sum_{i} \Delta V_{i}$ is the van der Waals volume of the repeating unit [9]; $A$ is a constant; $N_{A}$ is the Avogadro number. On the basis of Eq. (2) the $T_{C}$ value of the anthracene-containing homopolymer is determined as $514 \mathrm{~K}$.

In the third version of the calculation increments of the van der Waals volumes of the atoms $V_{i}$ and the $a_{i}$ and $b_{i}$ values [10] characteristic of each atom and each type of interaction were used:

$$
T_{c}=\frac{\sum_{i} \Delta V_{i}}{\sum_{i}\left(a_{i} \Delta V_{i}+b_{i}\right)} .
$$

Substitution of the necessary values in Eq. (3) gives $T_{c}=517 \mathrm{~K}$ for the anthracene-containing homopolymer. The average value of $T_{c}=513 \mathrm{~K}$ is significantly higher than the glass transition point for polymethylmethacrylate - the base of reoxane (378 K [6]). A photoneutral comonomer, the homopolymer of which has $T_{c}=293 \mathrm{~K}$, was used to lower the $T_{c}$ value. Its mole fraction of 80 mole $\%$ gives the calculated $T_{c}=338 \mathrm{~K}$ for the investigated polymeric material.

Methylene blue dye in ratios of 800:1, 1000:1, 1400:1, and 1700:1 by weight was used as sensitizer that generates singlet oxygen. The highest diffraction efficiency for recording the diffraction grids was obtained with the 1700:1 polymer/ dye ratio, and this was therefore used to study the rate of photooxidation. Photorecording layers with thickness of $>2 \mu \mathrm{m}$ were deposited by pouring a solution of the polymer and dye onto glass substrates. Thinner layers were obtained by centrifuging the solution also on the glass substrates. A light-emitting diode with $\lambda_{\max }=650 \mathrm{~nm}$ exposing the sample at $\sim 6 \mathrm{~mW} / \mathrm{cm}^{2}$ was used to determine the efficiency of photo-oxidation. The phototransformation of the anthracene groups was followed from their electronic absorption spectra in the region of 330-410 nm, recorded on a Specord M40 spectrophotometer. The relative concentration of residual anthracene structures, averaged over the layer thickness, was obtained from the spectral data for various exposure times. Their photo-oxidation rate in layers of material of various thicknesses was characterized by the time $t_{e}$ required for the average concentration to decrease by $e$ times. The photorefractive effect with uniform irradiation by the light-emitting diode was determined by measuring the refractive index of the layer of material $n$ before and after irradiation at $\lambda=633 \mathrm{~nm}$. For this, a technique based on measuring the waveguide refractive indices of the layer modes with a highly refractive prism pressed against its surface was used [11]. Transmitting holographic gratings with a period of $d=1.15 \mathrm{~mm}$ were recorded symmetrically with radiation from a He-Ne laser at $\lambda=633 \mathrm{~nm}$. The intensity of each of the laser beams during holographic recording was $\sim 0.5 \mathrm{~mW} / \mathrm{cm}^{2}$. The diffraction efficiency $\eta$ was calculated by means of the formula:

$$
\eta=\frac{I_{-1}}{I_{-1}+I_{0}}
$$

where $I_{0}$ is the zero-order beam intensity and $I_{-1}$ is the intensity of the diffraction beam behind the photographic plate while the grating was being recorded. 

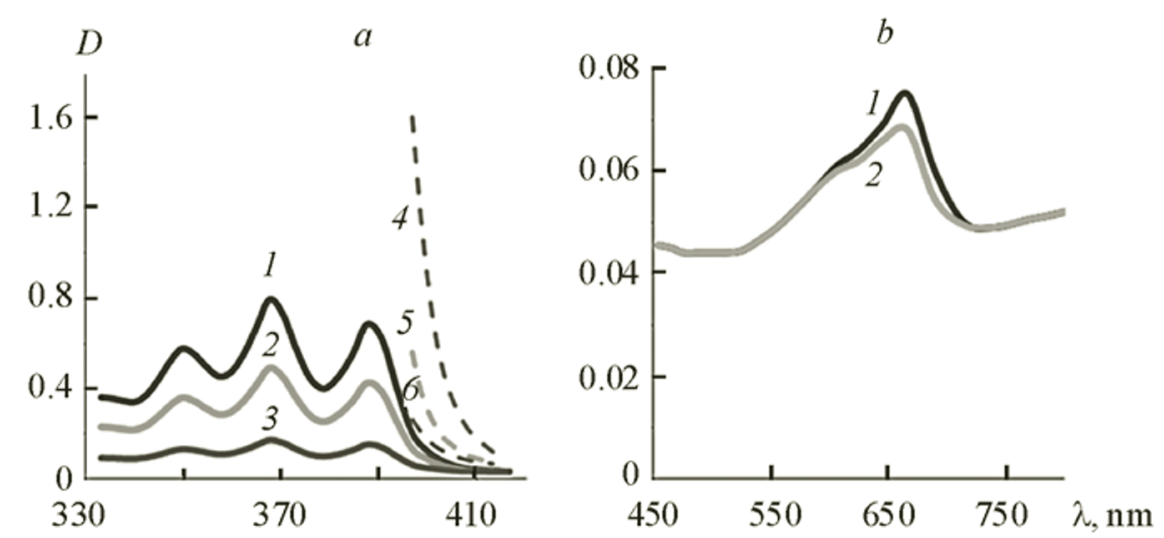

Fig. 1. Absorption spectra: a) anthracene groups in layers with thicknesses of $0.7(1-3)$ and $7.5 \mu \mathrm{m}(4-6)$ during LED irradiation for $0(1,4), 30(2,5)$, and $70 \mathrm{~min}(3,6)$; b) sensitizer dye in layer 7.5- $\mu \mathrm{m}$ thick before (1) and after exposure for $70 \mathrm{~min}(2)$.

Results and Discussion. Figure 1 shows the absorption spectra of the photosensitive layers for various LED irradiation times. The intensity of the long-wave structured band of the anthracene units for the thin layers decreases during irradiation (Fig. 1a, curves 1-3), tending toward zero values. This corresponds to the following scheme for the photoreaction:<smiles>[R]c1c2[c-][o+]ccc2cc2ccccc12</smiles>

which leads to disappearance of the unsaturated bonds in the central ring. For layers of significant thickness the photoreaction reduces the edge absorption (Fig. 1a, curves 4-6). At the same time some decrease in the absorption intensity of the sensitizer is observed (Fig. 1b, curves 1 and 2), indicating that its consumption is insignificant compared with the anthracene groups.

Increase of the thickness of the photosensitive layer must hinder diffusion saturation of the layer with oxygen and reduce the average quantum yield of the photoreaction $\beta$. Since their optical density in the spectral region of exposure is $\leq 0.2$ while the distribution of the intensity in the thickness is uniform, the layers can be considered optically thin over the whole range of thicknesses $(1-60 \mu \mathrm{m})$. Under these conditions and with unchanged concentration of oxygen over the whole volume of the material the time $t_{e}$ for decrease of the concentration of anthracene groups by $e$ times is determined by [1]:

$$
t_{e}=\frac{\left(C_{a}(0)-C_{a}\left(t_{e}\right)\right)}{I_{e} \sigma_{s} C_{s} \beta}=\frac{C_{a}(0)(1-e)}{e I_{e} \sigma_{s} C_{s} \beta},
$$

where $C_{a}(0)$ and $C_{a}\left(t_{e}\right)$ are the initial and residual (at time $t_{e}$ ) concentrations of anthracene; $I_{e}$ is the intensity of the radiation; $\sigma_{s}$ and $C s$ are the absorption section and the concentration of the sensitizer. Inverse proportionality between $t_{e}$ and $\beta$ and also invariability of the first value with invariability of the second follow from Eq. (5). Figure 2a shows the dependence of $t_{e}$ on the thickness of the layer $l$, which on the section up to 10-11 $\mu \mathrm{m}$ demonstrates the approximate constancy of $t_{e}$. With further increase of the thickness of the film there is an increase in the characteristic exposure time. The interpretation of this relationship is fairly obvious - with large thicknesses the diffusion of oxygen through the surface of the layer is not in a state to maintain a constant concentration of dissolved oxygen throughout the thickness, which leads to decrease of $\beta$ in the depth of the layer and to increase of the averaged values of $t_{e}$.

The refractive indices of the material at $\lambda=633 \mathrm{~nm}$ for the photosensitive layers $4-5-\mu \mathrm{m}$ thick were determined before $\left(n_{0}\right)$ and after uniform irradiation with the LED to $\sim 90 \%$ conversion of the anthracene groups $\left(n_{t}\right)$. The values $n_{0}=1.545$ and $n_{t}=1.525$ were identical for polarizations of the probing laser beam in the plane of and perpendicular to the layer (the TE and TM polarization of the waveguide modes of the layer excited during probing). The change of refractive index produced by irradiation was $\Delta n=n_{t}-n_{0}=-0.02$. For comparison Popov et al. [12] obtained a maximum value $\Delta n \sim-0.005(\lambda=633 \mathrm{~nm})$ for plates of one of the forms of reoxane 1-mm thick and previously saturated with oxygen. The 

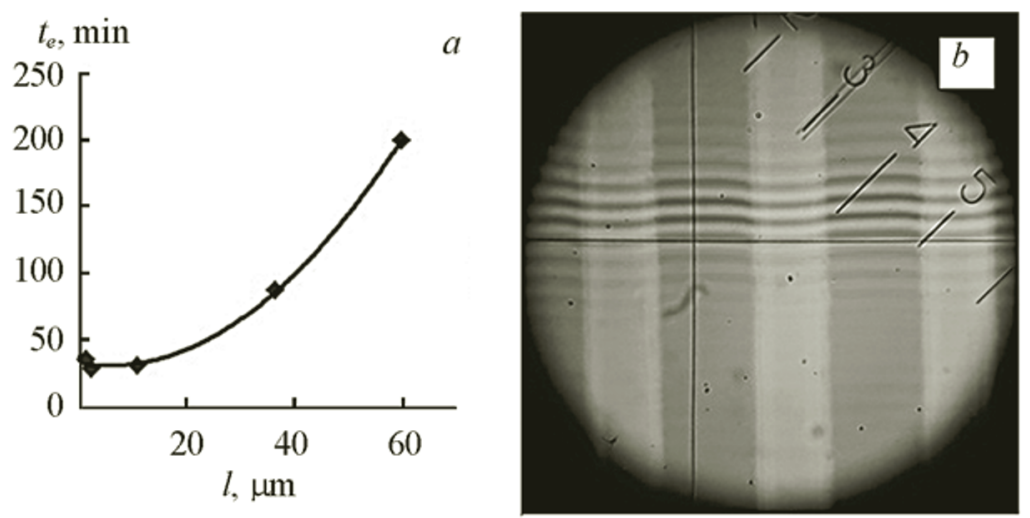

Fig. 2. Dependence of LED irradiation time $t_{e}$ required to reduce the average concentration of anthracene groups by a factor of $e$ on the layer thickness $l$ (a); the interference pattern in the MII-4 eyepiece during reflection of light from irradiated layer on a photo mask (b).

large value of $\Delta n$ may be due to the higher concentration of anthracene groups in the material ( $\sim 20$ mole $\%)$ resulting from their addition to the macromolecules. Evaluation of the sensitivity $S$ by the formula [12]:

$$
S=\Delta n / H_{e},
$$

where $H_{e}$ is the exposure dose during irradiation $\left(H_{e}=I_{e} t_{e}\right)$, gives $S=1.4 \cdot 10^{-3} \mathrm{~cm}^{2} / \mathrm{J}$, which is substantially higher than for reoxane $\left(1.6 \cdot 10^{-4} \mathrm{~cm}^{2} / \mathrm{J}[12]\right)$. Possible reasons for the increase of sensitivity under conditions of low oxygen concentration are the large concentration of anthracene groups and the high mobility of the oxygen molecules resulting from the lower glass transition temperature of the material. In addition to the above-mentioned factors the experimentally discovered expansion of the investigated material during irradiation can lead to increased sensitivity. In order to observe it a layer of the photosensitive material was deposited on the surface of a glass substrate with a deposited metal grating (period $70 \mu \mathrm{m}$ ) and irradiated with LED radiation on the substrate side. Figure $2 \mathrm{~b}$ shows a photograph of the interference pattern in an MII-4 eyepiece with reflection of light from the irradiated layer on a photo mask. The rise of the interference bands in the dark areas correspond to protrusions on the surface of areas of the layer that were illuminated during exposure. If the picture is interpreted as expansion of the layer resulting from phototransformation and it is considered to be unidimensional (perpendicular to the plane), the relative increase in thickness is then $\Delta l / l=-\Delta \rho / \rho \approx 0.004$. According to the following expression [13]:

$$
\Delta n \approx \frac{\left(n^{2}+2\right)\left(n^{2}-1\right)}{6 n} \frac{\Delta \rho}{\rho}
$$

such change of density will lead to change of refractive index $\Delta n \approx-0.0025$, i.e., expansion of the irradiation material under the specified conditions can make an appreciable contribution to the photorefraction effect. However, this contribution cannot explain the increased sensitivity of the material over the sensitivity of reoxane. The reason seems to be the higher rate of the photochemical reaction. Determination of the molecular volumes of the anthracene units and their peroxide by increments [9] gives 0.263 and $0.274 \mathrm{~nm}^{3}$. Increase in the molecular volume as a result of the photoreaction implies an increase in its efficiency with a larger fraction of free volume in the polymeric medium at a lower glass transition temperature. The higher accumulation rate of the photoproduct is also favored by the higher concentration of anthracene units compared with reoxane.

Holographic recording was used to determine the optimal proportion of sensitizer in polymer material. Figure $3 \mathrm{a}$ shows the kinetics of the diffraction efficiency in the holographic recording process in layers of material with various polymer/dye mass ratios and demonstrates the advantage of recording in a layer with a selected ratio of 1700:1. The reason for the reduced efficiency of recording with increased sensitizer concentration is probably its aggregation at high concentrations (Fig. 3b).

The volumetric holographic gratings were recorded with laser beams polarized in the vertical plane in layers 5-20- $\mu \mathrm{m}$ thick. Figure 4a shows a series of kinetics of the diffraction efficiency for layers with various thicknesses and 

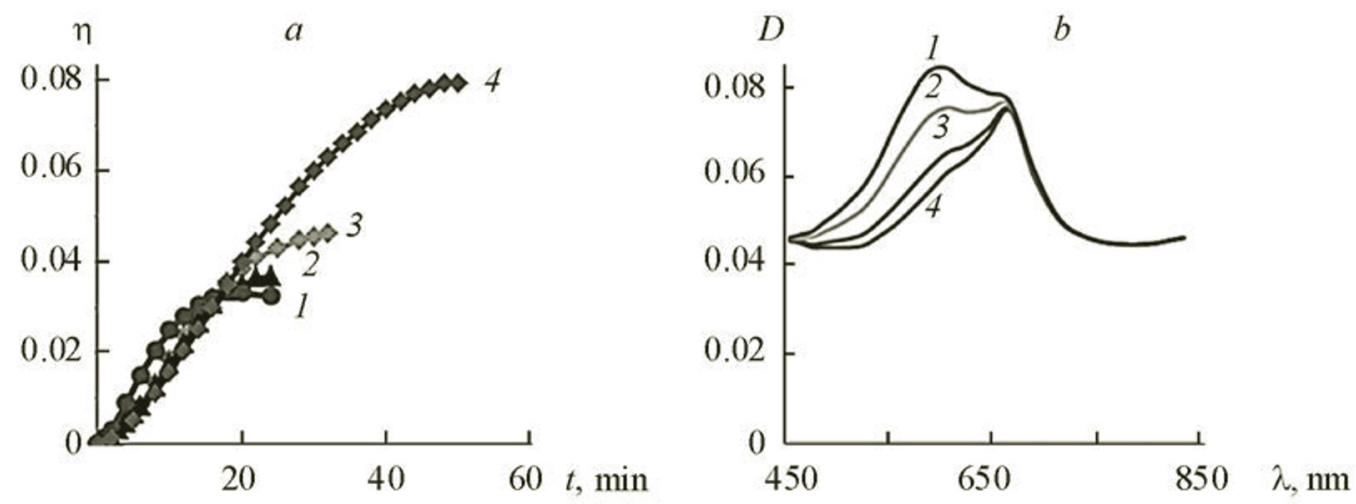

Fig. 3. Kinetics of diffraction efficiency in holographic recording (a) and electronic absorption of dye (b) for polymer/dye mass ratios 800:1 (1), 1000:1 (2), 1400:1 (3), and 1700:1 (4).

a polymer/dye ratio of 1700:1. The increase in the maximum values of the diffraction efficiency becomes slower with increasing thickness and becomes saturated when the thickness approaches $20 \mu \mathrm{m}$. The difference in optical thicknesses at the maxima and minima $\Delta=2 \Delta n_{1} l_{0}$ created in the structure of holographic gratings was calculated by means of the expression for the diffraction efficiency of volume phase holographic gratings:

$$
\eta=\sin ^{2} \frac{\pi \Delta n_{1} l_{0}}{\lambda \cos \theta}
$$

where $\Delta n_{1}$ is the amplitude of modulation of the refractive index; $l_{0}$ is the layer thickness; $\theta$ is the angle if incidence of the recording beams on the surface of the layer. The dependence of $\Delta$ on $l_{0}$ (Fig. $4 \mathrm{~b}$, curve 1 ) increases up to $\sim 10 \mu \mathrm{m}$, and it is then practically horizontal. There is no reason to consider that increase of the layer thickness to $>10 \mu \mathrm{m}$ changed its properties so that the amplitude $\Delta n_{1}$ began to change in inverse proportion to the thickness. A more probable assumption is that a holographic grating with an effective thickness smaller than the geometric thickness is formed in the thicker layers. The front boundary of the lattice is formed by the photooxidation front [14], while the appearance of the back boundary at a layer thickness of $>10 \mu \mathrm{m}$ can be attributed to increase in the root mean square displacement of the singlet oxygen molecules with increase in the photoconversion to values comparable with the lattice constant.

A refraction sphere with a radius $R_{S P}$ in the order of the root mean square displacement of singlet oxygen molecules, created during photo-oxidation around the sensitizer molecule, is regarded as a "grain" of the phase image in reoxane [1]. The radius was estimated by means of the equation:

$$
R_{S P} \sim \sqrt{6 D_{1_{\mathrm{O}_{2}}} \tau_{1_{1}}},
$$

where $\tau_{\mathrm{O}_{2}}$ and $D_{\mathrm{O}_{2}}$ are the life time and diffusion coefficient of singlet oxygen, which for reoxane is in the order of hundredths of a micrometer. A larger diffusion coefficient and a clearly defined dependence of the life time on the degree of photoconversion of the anthracene groups can be expected for the investigated material. A high concentration of the latter ( $\sim 2$ mole/L) must significantly reduce $\tau_{1}$ at the beginning of exposure on account of the loss of singlet oxygen on the anthracene fragments. During oxidation of the latter $\tau_{1} \mathrm{O}_{2}$ must increase, raising the radius of the photoreaction sphere. On the whole, this can lead to degradation of the holographic grating in the surface layer, which is most highly enriched with oxygen. As a result, the most effective grating exists in a region of limited thickness, moving into the depth of the recording layer during writing. Degradation of the lattice near the surface begins in layers with a thickness of $\sim 10 \mu \mathrm{m}$, which, within the framework of our qualitative model, corresponds to the effective lattice thickness in thicker layers (Fig. 4b, curve 1). If we divide the obtained values of $\Delta$ into doubled geometric thicknesses of the layers with $l_{0}<10 \mu \mathrm{m}$, and with $l_{0}>10 \mu \mathrm{m}$ by $20 \mu \mathrm{m}$, we obtain the amplitudes of modulation of the refractive index $\Delta n_{1}$, which are represented in Fig. $4 \mathrm{~b}$ by a number of points close to 0.006 (curve 2). The calculated $\Delta n_{1}$ value is approximately constant for the recording layers over the entire investigated range of thicknesses, and this provides a qualitative model for explaining the experimental dependence of the 

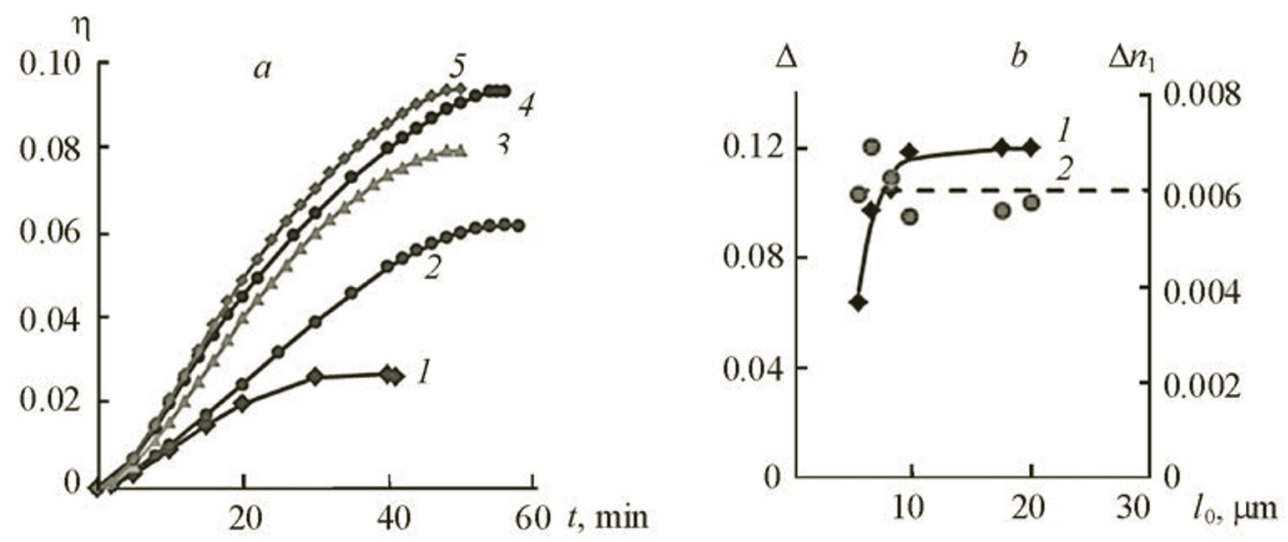

Fig. 4. Kinetics of recording of holographic gratings (a) with layer thicknesses of 5.4 (1), 6.4 (2), 8.1 and 9.8 (3), 17.6 (4), and $20.0 \mu \mathrm{m}$ (5); dependence of the path difference (1) and modulation amplitude of the refractive index (2) on the layer thickness (b) calculated for maximum diffraction efficiency; polymer/dye mass ratio 1700: 1 .

obtained diffraction efficiency on the thickness of the recording layer. The value of $2 \Delta n_{1} \approx 0.012$ amounts to $60 \%$ of the maximum possible total degree of modulation of the refractive index.

Conclusions. Possible volume phase recording by photo-oxidation of the anthracene side groups of polymer molecules by oxygen that enters the layer through the boundary with air was confirmed experimentally. It is shown that photo-oxidation under the action of light-emitting diode radiation with $\lambda_{\max }=650 \mathrm{~nm}$ at $6 \mathrm{~mW} / \mathrm{cm}^{2}$ in new polymeric material with addition of methylene blue as photosensitizer gives an almost constant quantum yield if the layer thickness does not exceed $10 \mu \mathrm{m}$. As a result of $\sim 90 \%$ photoconversion of the anthracene groups the refractive index of the material is reduced by 0.02 . The phototransformations are accompanied by increase of layer thickness as a result of increase in the volume of the molecular structure during photo-oxidation, and this can contribute to change of $n$ at a level of 0.002-0.003. Holographic gratings with a period of $1.15 \mathrm{~mm}$, recorded in layers $5-20-\mu \mathrm{m}$ thick, had maximum diffraction efficiency approaching $10 \%$. The kinetics of holographic recording was characterized by diffraction efficiency to saturation until maximum modulation depth of refractive index is reached long before overmodulation of the grating. The effect is explained by increase in the size of the photorefractive sphere with depth of photoconversion as a result of decreased probability of loss of singlet oxygen on the anthracene groups. This leads to a decrease of the modulation depth $n$, gradual erasure of the part of the grating closest to the surface of the layer during recording, and its effective thickness $e$ less than the geometric thickness of the recording layer.

\section{REFERENCES}

1. V. I. Sukhanov, G. I. Lashkov, A. E. Petnikov, Yu. V. Ashcheulov, I. I. Reznikova, and A. S. Cherkasov, Optical Holography [in Russian], Nauka, Leningrad (1979), pp. 21-42.

2. V. I. Sukhanov, Uspekhi Fiz. Nauk, 148, No. 3, 541-542 (1986).

3. G. I. Lashkov, Uspekhi Fiz. Nauk, 148, No. 3, 539-541 (1986).

4. V. I. Sukhanov, A. V. Veniaminov, A. I. Ryskin, and N. V. Nikonorov, Coll. All-Russia Seminar "Yurii Nikolaevich Denisyuk - Founder of National Holography" [in Russian], SPbGU-ITMO, St. Petersburg (2007), pp. 262-276.

5. A. V. Veniaminov, G. I. Lashkov, O. V. Ratner, N. S. Shelekhov, and O. V. Babdyuk, Opt. Spektrosk., No. 1, 142-147 (1986).

6. S. A. Rentlinger, Permeability of Polymeric Materials [in Russian], Khimiya, Moscow (1974).

7. A. A. Askadskii, Vysokomol. Soed., A9, No. 2, 418-432 (1967).

8. A. A. Askadskii and G. L. Slonimskii, Vysokomol. Soed., A13, No. 9, 1917-1919 (1971).

9. A. A. Askadskii, G. L. Slonimskii, and A. I. Kitaigorodskii, Vysokomol. Soed., A12, No. 3, 494-512 (1970).

10. A. A. Askadskii, G. L. Slonimskii, Yu. I. Matveev, and V. V. Korshak, Vysokomol. Soed., A18, No. 9, 2067-2074 (1976). 
11. U. V. Mahilny and A. I. Stankevich, KnE Energy \& Physics, 7-13 (2018); DOI: 10.18502/ken.v3i3.2008.

12. A. P. Popov, A. F. Kavtrev, A. V. Veniaminov, and G. I. Lashkov, Optical Holography with Recording in ThreeDimensional Media (Yu. N. Denisyuk (Ed.)), Nauka, Leningrad (1986), pp. 82-91.

13. V. V. Mogil'nyi, Polymeric Photorecording Materials and Their Application: Lecture Course [in Russian], BGU, Minsk (2003), p. 58.

14. P. A. Kondratenko and L. Ya. Tantsyura, Khim. Fizika, 4, No. 6, 843-850 (1985). 